\title{
Power Problems in VLSI Circuit Testing ${ }^{\star}$
}

\author{
Farhana Rashid ${ }^{\star \star}$ and Vishwani D. Agrawal \\ Auburn University \\ Department of Electrical and Computer Engineering \\ 200 Broun Hall, Auburn, AL 36849 USA \\ fzr0001@tigermail.auburn.edu, vagrawal@eng.auburn.edu \\ http://www. eng. auburn. edu/ vagrawal
}

\begin{abstract}
Controlling or reducing power consumption during test and reducing test time are conflicting goals. Weighted random patterns (WRP) and transition density patterns (TDP) can be effectively deployed to reduce test length with higher fault coverage in scan-BIST circuits. New test pattern generators (TPG) are proposed to generate weighted random patterns and controlled transition density patterns to facilitate efficient scan-BIST implementations. We achieve reduction in test application time without sacrificing fault coverage while maintaining any given test power constrain by dynamically adapting the scan clock, accomplished by a built-in hardware monitor of transition density in the scan register.
\end{abstract}

Keywords: Test power, test time, transition density, weighted random patterns, built-in self-test, scan testing.

\section{Introduction}

Controlling power dissipation in large circuits during test is a major concern in the VLSI industry. High power dissipation occurs during test because, unlike the normal mode operation of the system, correlations between consecutive test patterns do not exist in the test mode [6, 7]. To increase the correlation between consecutive vectors during testing, several techniques have been proposed for creating low transition density in pattern sets and thus control the power dissipation. However, this in turn increases the test application time as the test has to run for longer time to reach sufficient fault coverage. Increase in test time is also undesirable.

In this work we show that by properly selecting the characteristics of either weighted random patterns (WRP) or transition density patterns (TDP) for a given circuit we can reduce the test time that is proportional to the number of vectors. In some cases, we may also get higher fault coverage because many

\footnotetext{
* Research supported in part by the National Science Foundation Grants CNS-0708962 and CCF-1116213.

${ }^{\star \star}$ Present Address: Intel Corporation, 1501 S. Mo-Pac Expressway, Suite 400, Austin, TX 78746 USA, farhana.rashid@intel.com.
} 
random-pattern resistant faults become detectable by WRP or TDP. Section 3 describes experiments run on the ISCAS89 benchmark circuits and compares the test lengths to reach certain fault coverage for weighted random patterns and transition density patterns against the conventional "purely" random patterns.

New test pattern generators (TPG) proposed in Section 4 produce vectors with desired weights or transition densities. Section 5 shows that the test time can be further reduced while maintaining any given power constrain by using a dynamically adaptive scan clock [15, 16]. Section 6 reports simulation results.

\section{Background}

Weighted random patterns have been used before to reduce test length for combinational circuits $1[3,8]$. Proper selection of the input probability can increase the efficiency of test vectors in detecting faults, resulting in reduced test time [10]. Therefore, to achieve higher fault coverage with shorter test lengths weighted pseudo random patterns are used [5].

Weighted random patterns (WRP) in which the probability of $1, p 1$, instead of being 0.5 , can be set to any value in the range $[0,1]$ have certain advantages. Recent papers discuss low power test using weighted random and other reduced activity patterns. The power dissipation of scan patterns is related to the transitions they produce in the scan register. It is reported that with reduced activity patterns the fault coverage rises slowly and for the same required coverage a larger number of patterns are needed. Thus, a reduced power test may take longer time.

Much work has been reported on the generation and application of WRP in BIST and random testing since the 70s. We cite only a few references here [8, 10]. The primary purpose of WRP is to increase the rate of fault detection and reduce the test time. They are also known to reduce power consumption 22].

Transition density patterns (TDP) are primarily used for reducing power consumption during test [12, 20]. Their potential for enhancing the fault coverage, the main topic this paper, has not been explored before. Transition density for a signal or a circuit was originally defined for estimating the dynamic power as the number of signal transitions per unit time [11].

We consider built-in self-test of full-scan circuits. A hardware test pattern generator (TPG) feeds bits serially into the scan chain. A test controller switches between test and normal modes to perform test-per-scan of the combinational logic as scan-out response bits are sent serially to a signature analyzer (SA). Typical TPGs use a linear feedback shift register (LFSR) or cellular automata (CA) to generate equiprobable 0 s and $1 \mathrm{~s}$ [19].

In scan BIST when the circuit is clocked in the scan mode, the shifting of pattern in the scan register produces transitions that cause power consumption in flip-flops and the combinational circuit. We can call them source transitions. The number of source transitions per clock is the total number of transitions in the bit-stream held in the scan register. As the scan register is loaded during scan-in, the probability of transitions in the incoming bit-stream determines 
the average number of transitions that the register will contain. The scanned-in pattern is applied to the combinational logic during normal mode of a test-perscan process. We do not consider the activity caused by the capture bits on which the scan-in does not have a direct control. Thus, the probability of transitions in the scan-in bits defines the transition density patterns (TDP).

\section{Fault Coverage of WRP and TDP}

We experimentally examine the fault coverage capabilities of weighted random patterns and transition density patterns.

\subsection{Weighted Random Patterns}

A Matlab program was written to construct different test vector sets. Each set contained 10,000 vectors but with different weights. Here, the weight is defined as the probability $p 1$ of a bit being 1 in a vector. The weights are varied from 0.1 to 0.95 at 0.05 intervals. Thus, 18 sets of vectors are constructed for the weights $0.1,0.15,0.2$, etc., up to 0.95 .

Target fault coverage was set at $95 \%$ of the total faults and fault simulation was done using the 18 vector sets mentioned above. The number of vectors needed to reach the target fault coverage by each vector set was recorded. For every circuit that was simulated there exists one specific weight that resulted in the shortest test length. The number of vectors obtained in this experiment for s1269 circuit as a function of the weight (probability of 1 in the scan-in bits) is shown in Figure 1. For this circuit the minimum vectors required for achieving the $95 \%$ target fault coverage is 22 , obtained for weight $p 1=0.6$.

\subsection{Computing Best Case Transition Density from Best Case Weight}

The transition density of the best case weighted random patterns can be estimated. The transition density in an uncorrelated-bit sequence that has a 0 probability of $p 0$ and 1 probability of $p 1$ is given by $p 0 p 1+p 1 p 0$ since a transition occurs when a 1 follows a 0 or a 0 follows a 1 . However, $p 0=1-p 1$, thus, the transition density can be calculated as:

$$
T D=(1-p 1) p 1+p 1(1-p 1)=2 p 1(1-p 1)
$$

Hence, from Figure 1 for circuit s1269, if best case weighted random pattern has a 1-bit probability of 0.6 then the corresponding transition density will be $2 \times 0.6 \times 0.4=0.48$.

This implies that if a test vector set is constructed to have a transition density of 0.48 , then that vector set will generate an effective test for the circuit with shortest test length. In other words it can be assumed that a vector set of average transition density of 0.48 will result in detecting more faults with fewer vectors when compared to the numbers of vectors applied with transition densities higher or lower than 0.48 . 


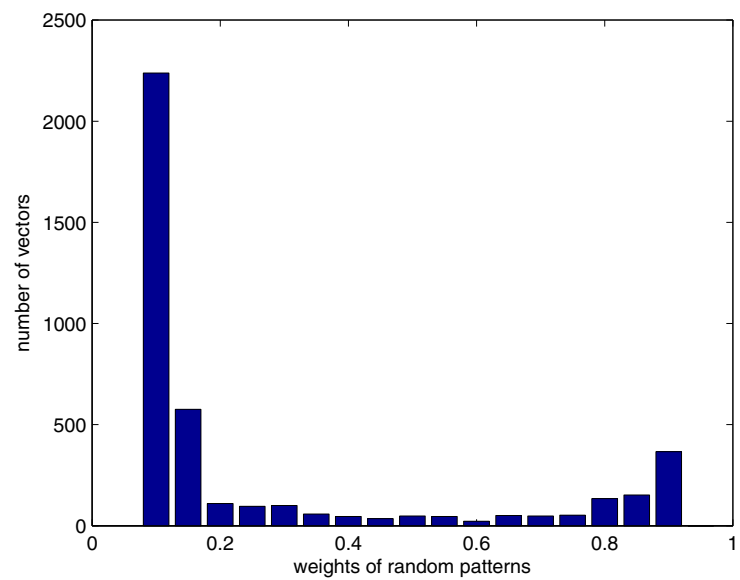

Fig. 1. Number of test-per-scan vectors for $95 \%$ coverage in s1269 when 1-probability $(p 1)$ of scan-in bits was weighted

\subsection{Transition Density Patterns}

If bits are generated randomly, the probabilities of generating a 1 or a 0 are equal, i.e., $p 0=p 1=0.5$. Hence the transition density of the bit stream is $2 \times p 0 \times p 1=$ 0.5 . To generate a transition density higher or lower than 0.5 , bits must be generated with negative or positive correlation, respectively. Therefore, the bit stream will contain shorter runs of consecutive $1 \mathrm{~s}$ or 0 s for a transition density higher than 0.5 and longer runs of consecutive $1 \mathrm{~s}$ or $0 \mathrm{~s}$ for a transition density lower than 0.5 .

A Matlab program was written to generate test vector sets, each containing 10,000 vectors but with different transition densities. Here also the transition density was varied from 0.1 to 0.95 , with 0.05 intervals. The vector set generated for 0.1 transition density has longer runs of $1 \mathrm{~s}$ and $0 \mathrm{~s}$ in consecutive bit positions. Likewise the vector set having transition density of 0.95 has very short runs of $1 \mathrm{~s}$ and $0 \mathrm{~s}$ in consecutive bit positions.

Target fault coverage was set to $95 \%$ of the total faults and then fault simulation was done using these 18 vector sets. In each case the number of vectors needed to reach the target fault coverage was recorded. For every circuit we simulated, there existed a best transition density $(T D)$ that resulted in the shortest test length. Figure 2 shows a bar chart of the number of transition density vectors obtained from fault simulation experiments to reach $95 \%$ fault coverage in circuit s1269. A vector set generated with 0.5 transition density has the best fault detecting capability with smallest number (only 24) as compared with the other transition density vector sets.

A set of ISCAS89 benchmark circuits was used for fault simulation with the transition density vector sets and weighted random vector sets. Table 1 shows the best case results obtained from fault simulation using AUSIM [18]. The table shows the numbers of vectors that achieved $95 \%$ fault coverage. The third column 


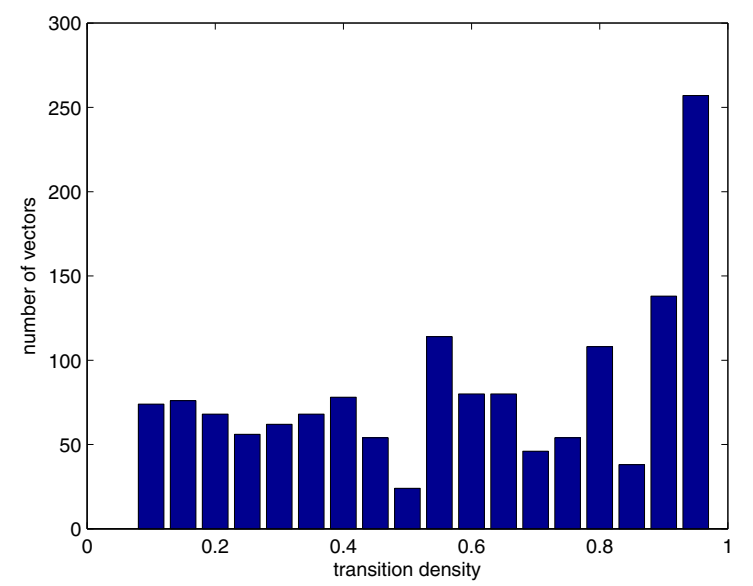

Fig. 2. Number of test-per-scan vectors for $95 \%$ coverage in s1269 for various transition densities of scan-in bits

Table 1. Best case weighted random and transition density vectors for $95 \%$ fault coverage in ISCAS89 circuits obtained from fault simulation of Matlab-generated patterns. Boldface numbers show the best choice for a circuit

\begin{tabular}{|c|c|c|c|c|c|c|}
\hline \multirow{2}{*}{$\begin{array}{c}\text { Circuit } \\
\text { name }\end{array}$} & Target & \multicolumn{3}{|c|}{ Weighted random vectors } & \multicolumn{2}{|c|}{ Transition density vectors } \\
\cline { 3 - 7 } & $p 1$ & No. of vectors & $T D=2 p 1(1-p 1)$ & Best $T D$ & No. of vectors \\
\hline \hline s298 & 77.1 & $\mathbf{0 . 6}$ & $\mathbf{1 8}$ & 0.48 & 0.55 & 423 \\
\hline s382 & 95 & $\mathbf{0 . 3}$ & $\mathbf{5 6}$ & 0.42 & 0.45 & 124 \\
\hline s510 & 95 & $\mathbf{0 . 4}$ & $\mathbf{1 3 6}$ & 0.48 & 0.5 & 152 \\
\hline s635 & 95 & $\mathbf{0 . 9}$ & $\mathbf{9 7}$ & 0.18 & 0.1 & 1883 \\
\hline s820 & 95 & $\mathbf{0 . 4 5}$ & $\mathbf{2 8 7 2}$ & 0.495 & 0.45 & 5972 \\
\hline s1196 & 95 & $\mathbf{0 . 5 5}$ & $\mathbf{1 7 0 6}$ & 0.495 & 0.45 & 2821 \\
\hline s1269 & 95 & $\mathbf{0 . 6}$ & $\mathbf{2 2}$ & 0.48 & 0.5 & 24 \\
\hline s1494 & 98.8 & 0.5 & 4974 & 0.5 & $\mathbf{0 . 4 5}$ & $\mathbf{3 1 5 8}$ \\
\hline s1512 & 95 & 0.75 & 538 & 0.375 & $\mathbf{0 . 2}$ & $\mathbf{3 3 8}$ \\
\hline
\end{tabular}

gives the weighted random bit probability $(p 1)$ that required minimum number of vectors shown in column 4 . In column 5 , the probability $p 1$ of column 3 is used to compute transition density from equation 1 .

The last two columns of Table 1 give the best case transition density $(T D)$ and the corresponding number of vectors obtained from simulation. The differences in the transition densities of columns 5 and 6 can be because the two were obtained from two different statistical test samples. Also, equation 1, used for computing $T D$ in column 5, assumes uncorrelated neighboring bits, an assumption that is yet to be validated for our transition density vectors.

However, unlike highly efficient weighted random patterns the patterns constructed based on transition density were not able to detect $100 \%$ of faults for 
some circuits. As shown in Table1, the weighted random patterns and the transition density based vectors do not always have the same effectiveness. Which is better, often depends upon the circuit. While the generation of weighted random patterns is well understood, transition density patterns need further study.

Note that weighted random bits have a transition density of their own. But our transition density patterns generated by the toggle flip-flop always have equal number of 0 s and 1s. Though the transition density of weighted random bits as obtained from equation 1 for any $p 1$ can never be higher than 0.5 , our transition density patterns generated by a toggle flip-flop (Section 4) can produce transition densities greater than 0.5 . Such patterns will produce high power consumption, which can be lowered by the adaptive test clock procedures [15] as discussed in a Section 5, if the vectors gave accelerated fault coverage. This aspect needs additional study.

\section{BIST-TPG for Controlled Weight Probability and Transition Density}

We propose a new test pattern generator (TPG) for producing vectors of desired weights or transition densities. The illustration in Figure 3 contains a 28-bit external linear feedback shift register (LFSR) using the polynomial $p(x)=x^{28}+$ $x^{3}+1$. The Scan Bit Generator block consists of AND gates, inverters, an 8-to-1 MUX to select from eight different probabilities of a bit being 1, and a toggle flip-flop. A simple finite state machine (FSM) provides the select inputs to the MUX. The Scan Bit Generator produces eight different weighted random bit sequences. The weights are constructed by ANDing two or more outputs from non-adjacent cells of the LFSR.

As shown in Figure 3, any one among eight weights for the probability $p 1$ of a bit being 1 , i.e., $0.125,0.25,0.375,0.4375,0.5,0.625,0.75$ and 0.875 , respectively, is selectable by an 8-to- 1 MUX. The probability of a bit being 1 or 0 at the output of any cell of the LFSR is 0.5 . These are signals W[0] through W[1]. One of these is directly fed to an input of the MUX. Two outputs from two non-adjacent cells were ANDed to produce a weight 0.25 , three outputs from three non-adjacent cells are ANDed to produce a weight 0.125, and inverting these two weights we get weights 0.75 and 0.875 , respectively. For generating a weight 0.375 , the weight 0.75 is again ANDed with another cell output that is not adjacent to any of those two cells that are used in creating the 0.75 weight. Similarly, for generating a weight 0.4375 , the weight 0.875 is ANDed with another non-adjacent cell output. Finally, to construct a weight 0.625 , the weight 0.375 is inverted. An FSM controls the three select lines of the 8-to-1 MUX to choose any intended probability $p 1$ for WRP_bits.

A toggle flip-flop constructed with a D flip-flop and an XOR gate produces bits with transition density $T D=p 1$ from the weighted random bits of weight $p 1$ as shown in Figure 3. Through the select lines of the MUX, weight $p 1$ is selected as the bit sequence fed to one of the inputs of the XOR gate; the other input line of the XOR gate is the output of the D flip-flop. The selected wight 


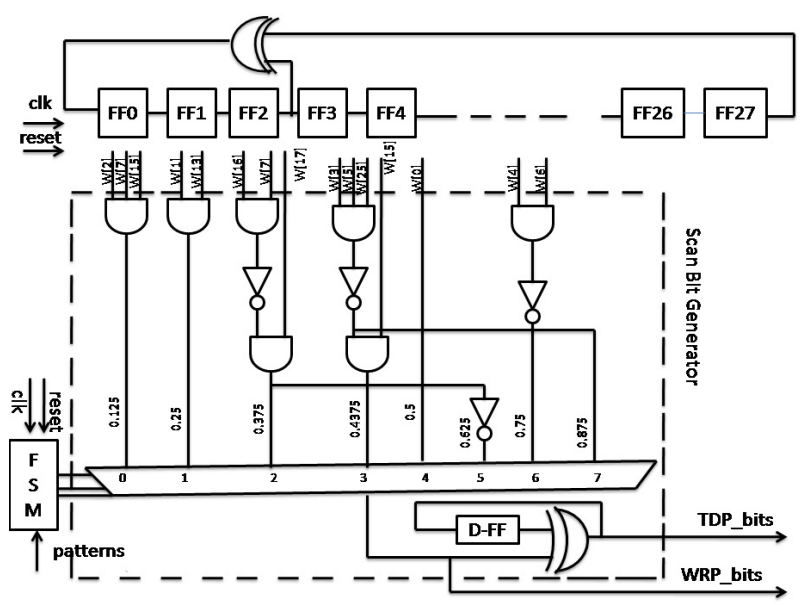

Fig. 3. Test pattern generator (TPG). Equiprobable $0-1$ bit outputs, W[0] through $\mathrm{W}[27$, of a 28-bit LFSR are transformed into weighted random pattern (WRP) and transition density pattern (TDP) bits for scan-in.

$p 1$ thus controls the transition density at the output of the XOR gate. A 1 in the bit sequence will produce a transition at the output of the XOR gate and a 0 will produce no transition. The resulting TDP_bits at the output of the XOR gate have a probability of a transition to occur, which is same as the weight $p 1$ selected from the MUX.

Output WRP_bits or TDP_bits from TPG of Figure 3 feeds the scan chain input of the circuit under test (CUT). For multiple scan chains, the Scan Bit Generator block in TPG is copied multiple times to generate inputs scan_in1, scan_2, etc., for scan chains, as discussed in the next section. The LFSR outputs, $\{\mathrm{W}[\mathrm{i}]\}$, are permuted differently as they are supplied to the duplicated blocks to reduce correlation among the scan chain inputs.

Figure 4 shows that the proposed TPG for WRP and TDP is capable of producing vectors with the desired weight $(p 1)$ or transition density. The bars in the figure show the numbers of TPG test-per-scan vectors for $95 \%$ fault coverage in s1512 as determined by fault simulation. The best cases are 406 TDP vectors for $T D=0.25$ and $768 \mathrm{WRP}$ vectors for $p 1=0.75$. These are within statistical variation from the data for s1512 in Table 1, which was obtained by fault simulation of Matlab-generated patterns. The best cases there were 338 TDP vectors for $T D=0.2$ and $538 \mathrm{WRP}$ vectors for $p 1=0.75$.

\section{Dynamic Control of Scan Clock in BIST Circuit with Modified TPG}

Recent work shows that by deploying a dynamic test clock control scheme in scan testing we can reduce test time while maintaining any given peak power limit $[14-17]$. We extend that technique to multiple scan chains and then use 


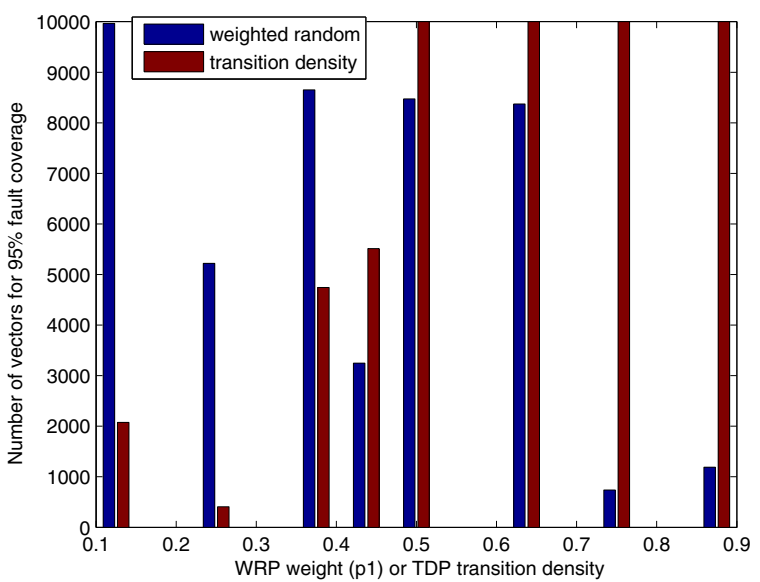

Fig. 4. Performance of transition density and weighted random patterns of s1512

the transition density or weighted random patterns produced by the TPG of the last section. The scheme automatically adjusts the scan clock to keep the test power constrained while reducing the test application time. Our objective is to examine the test time reduction benefits of various types of patterns.

The adaptive scheme of scan clock in scan-BIST consists of a separate inactivity monitor for each scan chain, which keeps track of total inactive bits entering the scan chain as shown in Figure 5 In addition, a frequency divider block provides different frequencies to choose from by a control clock select block. The scanning in of the bits of a test vector starts with the slowest test clock and depending on the number of inactive bits scanned in, the scan clock frequency is gradually increased. We assume that the captured vector produces worst-case activity of 1 , that is, the scan chains are filled with alternating $1 \mathrm{~s}$ and 0 s prior to scan-in. We use a TPG with multiple scan bit generator blocks as described in the previous section, along with a finite state machine that selects the weight or transition density from the TPG as shown in Figure 5. The finite state machine (FSM) takes the number of the patterns applied as inputs from the BIST controller and controls the wight or transition density of the test vectors.

The circuit under test (CUT) in Figure 5 has a built-in self-test (BIST) architecture with flip-flops inserted on all primary inputs (PI) and primary outputs (PO). All flip-flops are configured into multiple (e.g., four, as shown in the figure) scan chains of nearly equal lengths [13, 15]. Unlike the Illinois scan [9] where identical bits are broadcast to all chains, this TPG supplies (presumed, though not verified) independent bits, scan_in1 through scan_in4, to CUT, which in the figure has four scan chains. Under the control of a BIST controller once, using the scan mode, all chains are filled with bits from TPG, one normal mode clock cycle captures the circuit response in flip-flops. Then, again using the scan mode 


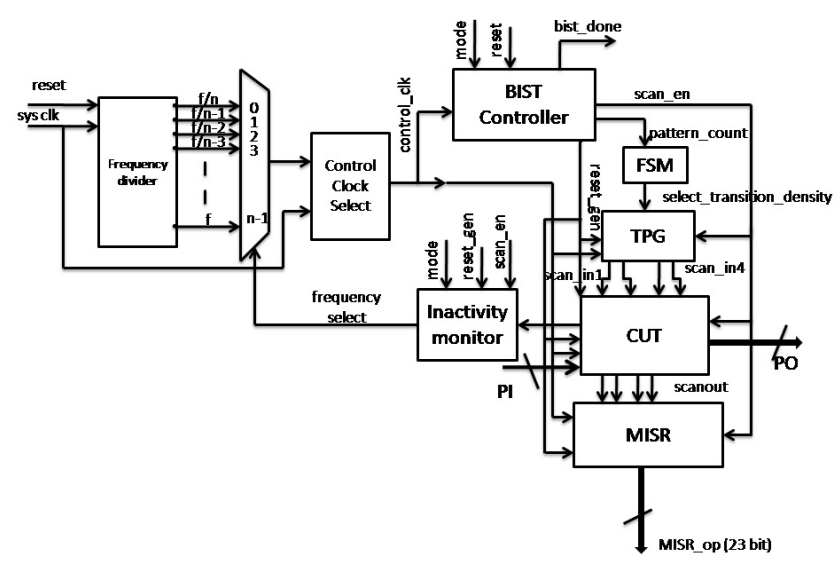

Fig. 5. Adaptive scan clock scheme with modified TPG

as the next pattern is scanned in the captured bits are supplied to a multiinput signature register (MISR) [4, 19]. This completes the application of one test-per-scan vector.

We can pre-determine the best case transition density with the modified TPG and run the whole test session with a pre-selected transition density. Also the circuitry to dynamically adapt the scan clock will help speed up the test clock by monitoring the inactivity and, therefore, keep the whole test session powerconstrained. The time reduction in scan-in will be dominated by the largest scan chain and for a transition density $T D$ and the number of frequencies available to adapt from $v$, the reduction in scan-in time is given by [13, 15],

$$
\text { Test time reduction }=\frac{1}{2}(1-T D)-\frac{1}{2 v}
$$

\section{Experimental Results}

Experiments were done on ISCAS89 benchmark circuits. Table 2 shows the comparison between the number of vectors needed to reach $90 \%$ fault coverage for each of the circuits. It is to be noted that for each circuit there exists a particular weight or a particular transition density that results in the shortest test length. Consider s13207 circuit with the conventional BIST using a fixed frequency clock. For a $90 \%$ target fault coverage, Table 2 gives 4262 random $(p 1=0.5)$ vectors, $2127 \mathrm{WRP}$ $(p 1=0.35)$ and 1490 TDP $(T D=0.3)$. We use the conventional BIST with random vectors as the reference for test time. When the BIST is implemented with TDP, the test time will be reduced by $100 \times(4262-1490) / 4262=65 \%$. There will be additional gain with adaptive clock.

So, we examine the reduction in test application time when a dynamic scan clock scheme is used [15]. Results for a selected set of ISCAS89 circuits are given 
Table 2. Test lengths for random and best-case weighted random (WRP) and transition density (TDP) patterns for $90 \%$ fault coverage in ISCAS 89 circuits

\begin{tabular}{|c|c|c|c|c|c|c|c|c|c|}
\hline Circuit & DFFs & Gates & \begin{tabular}{|l|l|} 
PIs & \\
\end{tabular} & POs & \multicolumn{3}{|c|}{ Number of vectors for $90 \%$ coverage } & $\begin{array}{c}p 1 \text { for } \\
\text { best WRP }\end{array}$ & $\begin{array}{c}T D \text { for } \\
\text { best TDP }\end{array}$ \\
\hline$\overline{\mathrm{s} 27}$ & $\overline{\overline{3}}$ & $\overline{10}$ & $\overline{\overline{4}}$ & $\overline{1}$ & $\overline{\overline{15}}$ & $\overline{\overline{5}}$ & $\overline{\overline{12}}$ & $\overline{0.15}$ & $\overline{0.6}$ \\
\hline s298 & 14 & 119 & 3 & 6 & 52 & 52 & $>10000$ & 0.5 & 0.0 \\
\hline s382 & 21 & 158 & 3 & 6 & 42 & 30 & 16 & 0.3 & 0.4 \\
\hline s386 & 6 & 159 & 7 & 7 & 621 & 317 & 609 & 0.35 & 0.4 \\
\hline s344 & 15 & 160 & 9 & 11 & 50 & 34 & $>10000$ & 0.45 & 0.0 \\
\hline s510 & 6 & 211 & 19 & 7 & 70 & 84 & 76 & 0.4 & 0.5 \\
\hline $\mathrm{s} 420$ & 16 & 218 & 18 & 1 & $>10000$ & 300 & 263 & 0.9 & 0.1 \\
\hline $\begin{array}{l}\mathrm{s} 832 \\
\end{array}$ & 5 & 287 & 18 & 19 & 1380 & 1126 & 2128 & 0.45 & 0.55 \\
\hline s820 & 5 & 289 & 18 & 19 & 962 & 806 & 1524 & 0.45 & 0.45 \\
\hline s641 & 19 & 379 & \begin{tabular}{|l|}
35 \\
\end{tabular} & 24 & 38 & 31 & 41 & 0.7 & 0.55 \\
\hline s713 & 19 & 393 & \begin{tabular}{|l|}
35 \\
\end{tabular} & 23 & 109 & 131 & 139 & 0.75 & 0.55 \\
\hline s967 & 29 & 394 & \begin{tabular}{|l|}
16 \\
\end{tabular} & 23 & 746 & 268 & 656 & 0.4 & 0.45 \\
\hline s953 & 29 & 395 & \begin{tabular}{|l|}
16 \\
\end{tabular} & 23 & 746 & 312 & 458 & 0.4 & 0.35 \\
\hline s838 & 32 & 446 & \begin{tabular}{|l|}
34 \\
\end{tabular} & 1 & $>10000$ & 488 & 491 & 0.95 & 0.15 \\
\hline s1238 & 18 & 508 & \begin{tabular}{|l|}
14 \\
\end{tabular} & 14 & 2506 & 1759 & 2598 & 0.55 & 0.45 \\
\hline s991 & 19 & 519 & 65 & 17 & 34 & 34 & 23 & 0.5 & 0.75 \\
\hline s1196 & 18 & 529 & 14 & 14 & 675 & 537 & 712 & 0.65 & 0.3 \\
\hline s1269 & 37 & 569 & 18 & 10 & 14 & 10 & 12 & 0.6 & 0.85 \\
\hline s1494 & 6 & 647 & 8 & 19 & 460 & 416 & 266 & 0.6 & 0.4 \\
\hline s1488 & 6 & 653 & 8 & 19 & 438 & 410 & 247 & 0.6 & 0.55 \\
\hline s1423 & 74 & 657 & \begin{tabular}{|l|}
17 \\
\end{tabular} & 5 & 12 & 6 & 11 & 0.85 & 0.2 \\
\hline s1512 & 57 & 780 & \begin{tabular}{|l|}
29 \\
\end{tabular} & 21 & 46 & 46 & 78 & 0.5 & 0.6 \\
\hline \begin{tabular}{|l|} 
s13207 \\
\end{tabular} & 669 & 7951 & \begin{tabular}{|l|}
31 \\
\end{tabular} & 121 & 4262 & 2127 & 1490 & 0.35 & 0.3 \\
\hline \begin{tabular}{|l|} 
s15850 \\
\end{tabular} & 597 & 9772 & 14 & 87 & 2463 & 2463 & 3293 & 0.5 & 0.3 \\
\hline
\end{tabular}

in Table 3. In each case four scan chains of nearly equal length were inserted using the BIST architecture of Figure 5. Test times for all three types of vectors shown in Table 2 were obtained by simulation.

As an example, consider s13207 again. Since flip-flops are added to primary inputs (PIs) and primary outputs (POs), total scanned flip-flops are $669+31+$ $121=821$. Given there are four nearly equal scan chains, the longest chain has $\lceil 821 / 4\rceil=206$ flip-flops. For fixed frequency test a clock period of 40ns is assumed. This is generally specified on the basis of the maximum energy consumption by a vector and the power dissipation capability of the circuit. Test time for 4262 random vectors is calculated as [4],

$$
\text { Test time }=(207 \times 4262+206) \times 40 \mathrm{~ns}=35.3 \mathrm{~ms}
$$

Similarly, the test time for 2127 WRP vectors is $17.6 \mathrm{~ms}$ and that for 1490 TDP vectors is $12.3 \mathrm{~ms}$. For this circuit, the shortest vector set is for TDP, which reduces the test time to $100 \times(35.3-12.3) / 35.3=0.65 \%$ of the random vector test time.

For the adaptive scheme, we use four clocks ( $v=4$ ) of periods 40ns, 30ns, 20ns and 10ns. Assuming the worst case activity from the captured bits, we begin each scan with the slowest clock of 40ns, which is sped up by the activity monitoring circuitry. Test times for the three types of vectors were obtained by simulation of the BIST circuit as $31.6 \mathrm{~ms}, 16.2 \mathrm{~ms}$ and $10.2 \mathrm{~ms}$, respectively, as shown in Table 3. When we compare the adaptive clock BIST with the best transition 
Table 3. Comparing test times for $90 \%$ coverage by conventional random (R), weighted random (WRP) and transition density (TDP) patterns when adaptive scan clock is used

\begin{tabular}{|c|c|c|c|c|c|}
\hline \multirow{2}{*}{ Circuit } & \multicolumn{5}{|c|}{ Types of patterns } \\
\cline { 3 - 6 } & $\begin{array}{c}\text { Random }(\mathrm{R}), p 1=0.5 \\
\text { test time (ns) }\end{array}$ & Weighted random (WRP) & Transition density (TDP) \\
\cline { 3 - 6 } & 10050 & 0.5 & 10050 & 0.5 & 1974026 \\
\hline \hline s298 & 10320 & 0.3 & 6661 & 0.4 & 8287 \\
\hline s382 & 18200 & 0.4 & 19570 & 0.4 & 19852 \\
\hline s510 & 348392 & 0.4 & 268971 & 0.4 & 504453 \\
\hline s820 & 418073 & 0.4 & 162371 & 0.3 & 231833 \\
\hline s953 & 264652 & 0.6 & 221416 & 0.3 & 262350 \\
\hline s1196 & 124572 & 0.6 & 117901 & 0.5 & 72831 \\
\hline s1488 & 31565011 & 0.35 & 16180025 & 0.3 & 10149712 \\
\hline s13207 & 16341260 & 0.5 & 16341260 & 0.3 & 20109065 \\
\hline s15850 & & & & & Best $T D$ \\
\hline
\end{tabular}

density patterns and the conventional fixed clock random pattern BIST, the test time reduction is $100 \times(35.3-10.2) / 35.3=71 \%$.

Once again, time reduction is measured as the time required for the vectors to be scanned-in using fixed scan clock minus the time required for the vectors to be scanned-in using the variable scan-clock. We see a significant reductions in test application time in s13207 and s1488 as the best case transition density vectors were applied along with the dynamic adaptive scan clock scheme.

\section{Conclusion}

For scan-BIST testing it is important to note that both power and test time contribute to the test cost as well as quality of the test. Transition density can be effectively selected for any circuit analogous to weighted random patterns to generate test session with shorter test length. Table 2 shows that for certain circuits, $90 \%$ fault coverage can be achieved with a minimal number of vectors if transition density patterns are used. Once the transition density is known the test application time can be further reduced by dynamically controlling the test clock keeping the test power under control as shown in Table 3. Thus, this work contributes towards generating high quality tests with reduced test application time and keeping the test power constrained.

Renewed interest in low power test patterns [12, 20, 21] has shown applications of low toggle rate vectors for reducing test power. Low toggle rate is often associated with slow rise in fault coverage. In this paper, we show that this is not necessarily true when the toggle rate (or transition density) is suitably determined for the circuit under test. Even higher toggle rates can be used when they provide quicker fault coverage because power consumption can be constrained by an adaptive scan clock, thus reducing the overall test time on balance. 


\section{References}

1. Agrawal, P., Agrawal, V.D.: On Improving the Efficiency of Monte Carlo Test Generation. In: Digest of 5th International Fault Tolerant Computing Symp., Paris, France, pp. 205-209 (June 1975)

2. Agrawal, P., Agrawal, V.D.: Probabilistic Analysis of Random Test Generation Method for Irredundant Combinational Networks. IEEE Trans. Computers C-24, 691-695 (1975)

3. Agrawal, P., Agrawal, V.D.: On Monte Carlo Testing of Logic Tree Networks. IEEE Trans. Computers C-25, 664-667 (1976)

4. Bushnell, M.L., Agrawal, V.D.: Essentials of Electronic Testing for Digital, Memory and Mixed-Signal VLSI Circuits. Springer (2000)

5. Eichelberger, E.B., Lindbloom, E.: Random-Pattern Coverage Enhancement and Diagnosis for LSSD Logic Self-Test. IBM Jour. Research and Development 27(3), 265-272 (1983)

6. Girard, P.: Low Power Testing of VLSI Circuits: Problems and Solutions. In: Proc. First IEEE Symp. Quality Electronic Design (ISQED), pp. 173-179 (March 2000)

7. Girard, P.: Survey of Low-Power Testing of VLSI Circuits. IEEE Design \& Test of Computers 19(3), 80-90 (2002)

8. Hartmann, J., Kemnitz, G.: How to Do Weighted Random Testing for BIST. In: Proc. IEEE/ACM International Conf. Computer-Aided Design, pp. 568-571 (November 1993)

9. Hsu, F., Butler, K., Patel, J.H.: A Case Study on the Implementation of Illinois Scan Architecture. In: Proc. International Test Conf., pp. 538-547 (2001)

10. Majumder, A.: On Evaluating and Optimizing Weights for Weighted Random Pattern Testing. IEEE Trans. Computers 45(8), 904-916 (1996)

11. Najm, F.: Transition Density: A New Measure of Activity in Digital Circuits. IEEE Trans. CAD 12, 310-323 (1993)

12. Rajski, J., Tyszer, J., Mrugalski, G., Nadeau-Dostie, B.: Test Generator with Preselected Toggling for Low Power Built-In Self-Test. In: Proc. 30th IEEE VLSI Test Symp., pp. 1-6 (April 2012)

13. Rashid, F.: Controlled Transition Density Based Power Constrained Scan-BIST with Reduced Test Time. Master's thesis, Auburn University, Alabama, USA (May 2012); A talk based on this thesis was presented in a student forum at the 21st IEEE North Atlantic Test Workshop, May 10 (2012)

14. Shanmugasundaram, P.: Test Time Optimization in Scan Circuits. Master's thesis, Auburn University, Alabama, USA

15. Shanmugasundaram, P., Agrawal, V.D.: Dynamic Scan Clock Control for Test Time Reduction Maintaining Peak Power Limit. In: Proc. 29th IEEE VLSI Test Symp., pp. 248-253 (May 2011)

16. Shanmugasundaram, P., Agrawal, V.D.: Dynamic Scan Clock Control in BIST Circuits. In: Proc. Joint IEEE Int. Conf. on Industrial Electronics and 43rd Southeastern Symp. on System Theory, pp. 237-242 (March 2011)

17. Shanmugasundaram, P., Agrawal, V.D.: Externally Tested Scan Circuit with BuiltIn Activity Monitor and Adaptive Test Clock. In: Proc. 25th International Conf. VLSI Design, pp. 448-453 (January 2012)

18. Stroud, C.E.: AUSIM - Auburn University SIMulator, http://www.eng. auburn.edu/users/strouce/ausim.html (accessed on March 6, 2012)

19. Stroud, C.E.: A Designer's Guide to Built-In Self-Test. Springer (2002) 
20. Tehranipoor, M., Nourani, M., Ahmed, N.: Low Transition LFSR for BISTBased Application. In: Proc. 14th IEEE Asian Test Symposium, pp. 138-143 (December 2005)

21. Udavanshi, S.: Design of Low Power and High Fault Coverage Test Pattern Generator for BIST. Master's thesis, Thaper University, Patiala, India (July 2011)

22. Wang, S.: Generation of Low Power Dissipation and High Fault Coverage Patterns for Scan-Based BIST. In: Proc. International Test Conf., pp. 834-843 (2002) 